\title{
CXCR4 Regulates Interneuron Migration in the Developing Neocortex
}

\author{
Ralf K. Stumm, ${ }^{1}$ Chun Zhou, ${ }^{1}$ Toshiaki Ara, ${ }^{2}$ Françoise Lazarini, ${ }^{3}$ Monique Dubois-Dalcq, ${ }^{3}$ Takashi Nagasawa, ${ }^{2}$ \\ Volker Höllt, ${ }^{1}$ and Stefan Schulz ${ }^{1}$ \\ ${ }^{1}$ Department of Pharmacology and Toxicology, Otto-von-Guericke University, 39120 Magdeburg, Germany, ${ }^{2}$ Department of Medical System Control, \\ Institute for Frontier Medical Sciences, Kyoto University, Kyoto 606-8507, Japan, and ${ }^{3}$ Unité de Neurovirologie et Régéneration du Système Nerveux, \\ Department of Neuroscience, Institut Pasteur, 75724, Cedex 15, Paris, France
}

The chemotactic factors directing interneuron migration during cerebrocortical development are essentially unknown. Here we identify the CXC chemokine receptor 4 (CXCR4) in interneuron precursors migrating from the basal forebrain to the neocortex and demonstrate that stromal cell-derived factor-1 (SDF-1) is a potent chemoattractant for isolated striatal precursors. In addition, we show that CXCR4 is present in early generated Cajal-Retzius cells of the cortical marginal zone. In mice with a null mutation in CXCR4 or SDF-1, interneurons were severely underrepresented in the superficial layers and ectopically placed in the deep layers of the neocortex. In contrast, the submeningeal positioning of Cajal-Retzius cells was unaffected. Thus, our findings suggest that SDF-1, which is highly expressed in the embryonic leptomeninx, selectively regulates migration and layer-specific integration of CXCR4-expressing interneurons during neocortical development.

Key words: CXC chemokine; stromal cell-derived factor-1; CXCR4; cerebral cortex; development; reelin; Cajal-Retzius cell; hippocampus; interneuron migration; immunocytochemistry; in situ hybridization

\section{Introduction}

Unraveling the molecular mechanisms of cerebrocortical layering is essential for our understanding of cortical function and dysfunction. Several factors including extracellular or transmembrane glycoproteins and integrins have been implicated in neuronal migration. In addition, $\alpha$ chemokines are emerging key players in telencephalic and cerebellar patterning. Indeed, deletion of the genes encoding for stromal cell-derived factor-1 (SDF-1) or its receptor CXC chemokine receptor 4 (CXCR4) is lethal for mice soon after birth, with severe abnormalities affecting many organs, including the hematopoietic system, cardiovascular system, and brain (Nagasawa et al., 1996; Tachibana et al., 1998; Zou et al., 1998). SDF-1 is a potent chemoattractant for leukocytes, germ cells, and neuronal precursors (Tashiro et al., 1993; Bleul et al., 1996; Lazarini et al., 2000; Doitsidou et al., 2002). SDF-1 is highly expressed in the leptomeninx and the major attractant for external germinal layer cells in the developing cerebellum (Klein et al., 2001; Lu et al., 2001; Tham et al., 2001; Zhu et al., 2002). In mice lacking SDF-1 or CXCR4, migration of granule cell precursors out of the external germinal layer occurs prematurely, resulting in abnormal development of the cerebellum (Ma et al., 1998). The migratory stream of granule cell

Received Jan. 22, 2003; revised Jan. 22, 2003; accepted March 13, 2003.

This work was supported by Volkswagen Stiftung Grant I/75172 (S.S.) and European Community Grant QLG3-CT2000-00911 (M.D.D.). We thank Dr. Carola Haas and Dr. Michael Frotscher (Institute of Anatomy, University of Freiburg, Germany) for kindly providing reelin CDNA. We also thank Dr. André Goffinet (Louvain Medical School, Brussels, Belgium) for kindly providing reelin G10 antibody, and Dr. Baleux (Pasteur Institute, Paris, France) for the normal and truncated SDF-1. Excellent technical assistance by Diana Gericke, Dana Mayer, Anke Reichenauer, Karina Schäfer, and Nancy Schlawin is gratefully acknowledged.

Correspondence should be addressed to Stefan Schulz, Institut für Pharmakologie und Toxikologie, Otto-vonGuericke-Universität Magdeburg, Leipziger Strasse 44, 39120 Magdeburg, Germany. E-mail: Stefan.Schulz@ medizin.uni-magdeburg.de.

Copyright $\odot 2003$ Society for Neuroscience $\quad$ 0270-6474/03/235123-08\$15.00/0 precursors from the primary germinal zone toward the dentate gyrus also depends on SDF-1/CXCR4 signaling (Bagri et al., 2002; Lu et al., 2002).

Given the involvement of CXCR4 in morphogenesis of the dentate gyrus and cerebellum, we hypothesized that meningealderived SDF-1 regulates pia-directed neuronal migration in the developing neocortex. At least three distinct modes of radial migration can be distinguished in the cortex, depending on the neuronal subtype (Nadarajah and Parnavelas, 2002). The first neurons to be generated are preplate neurons, which are thought to migrate through somal translocation (Nadarajah et al., 2001). Subsequently generated neurons form the cortical plate, which divides the preplate into subplate and marginal zone (MarinPadilla, 1998). Cajal-Retzius (CR) cells are a subpopulation of preplate neurons. They become selectively localized in the marginal zone, and via secretion of the extracellular matrix protein reelin they regulate migration of later born neurons (Frotscher, 1998; Super et al., 1998, 2000). Among these are pyramidal cells, which use glial guidance for inside-out locomotion from the cortical ventricular zone to the marginal zone. A large proportion of cerebrocortical interneurons originate from the subcortical ganglionic eminences and reach the deep portion of the cortex by tangential migration. Subsequently these neurons move radially toward the leptomeninx and integrate into the cortical plate (Marin and Rubenstein, 2001).

We previously demonstrated a role for SDF-1/CXCR4 in adaptive neuronal plasticity and cerebral leukocyte recruitment after adult brain lesion (Stumm et al., 2002). Here we provide the first detailed analysis of CXCR4 expression in the developing forebrain. We identify CXCR4 in migratory and differentiating neocortical interneurons as well as CR cells. The distribution of interneurons in the neocortex of SDF-1- and CXCR4-deficient 
mice is severely perturbed, whereas the localization of CR cells and projection neurons is unaffected. Our findings indicate that SDF-1 and CXCR4 are key signaling molecules guiding migration of distinct neuronal subtypes during late but not early cerebrocortical development.

\section{Materials and Methods}

Animals. Pregnant Wistar rats were obtained from Dimed Schönwalde (Schönwalde, Germany). Noon of the day on which vaginal plug was observed was termed embryonic day (E) 0.5 ; the day of birth was termed postnatal day $(\mathrm{P}) 0$. In situ hybridization analysis was performed in three rats at E13.5, E14.5, E15.5, E17.5, E18.5, E19.5, P0, P1, P3, P6, P9, P12, P16, P21, P31, and 6 weeks after birth (adult) using cryostat sections (14 $\mu \mathrm{m}$ ) after standard pretreatment (Stumm et al., 2002). For free-floating immunohistochemistry, postnatal animals were transcardially perfused (Stumm et al., 2002). Embryonic brains were immersion-fixed in BouinHollande (Stumm et al., 2001). Wild-type, SDF-1 ${ }^{+/-}$, CXCR4 ${ }^{+1-}$, SDF- $1^{-1-}$, and CXCR $4^{-1-}$ mice were obtained from breeding of heterozygous mice. Genotyping of the embryos has been described previously (Nagasawa et al., 1996; Ma et al., 1998). C57BL/6 mice used for the chemotaxis assay were purchased from IFFA CREDO (L'Arbresle, France). For all animal procedures, ethical approval was sought before the experiments according to national and institutional requirements.

In situ hybridization and immunohistochemistry. Procedures and cDNAs to generate the riboprobes for SDF-1 and CXCR4 have been described previously (Stumm et al., 2001, 2002). The SDF-1 probe is directed against the coding region and detects all rat SDF-1 isoforms. The $1.2 \mathrm{~kb}$ reelin cDNA (Haas et al., 2000) was generously provided by Dr. M. Frotscher (Freiburg, Germany). The coexpression of reelin and glutamic acid decarboxylase (GAD) with CXCR4 was analyzed by combined radioactive and nonradioactive in situ hybridization as described (Stumm et al., 2002). For analysis of proportional coexpression of CXCR4 and reelin mRNAs, 100 single- and double-labeled neurons were counted from three animals per developmental stage (E15.5, P0, P3, P6, P9, P12, P21, P31). Coexpression of GAD and CXCR4 was analyzed at P6, P12, and P21. The previously characterized affinity-purified rabbit antiserum $\{2144\}$ directed against the COOH-terminal tail of CXCR4 and the G10 mouse monoclonal antibody directed against reelin (generously provided by Dr. A. Goffinet, Louvain Medical School, Brussels,Belgium) were combined for dual immunofluorescence (de Bergeyck et al., 1997; Stumm et al., 2002). Slide-mounted immunohistochemistry of embryonic brains was performed after paraffin embedding according to standard protocols (Stumm et al., 2001). The density of CXCR4- and reelinexpressing neurons in different layers of the cortex was determined in SDF-1-deficient mice $(n=3)$. For each animal, 20 identically sized rectangular areas were tangentially positioned to the surface of the neocortex, and cell counts were performed and averaged. Statistical analysis was performed using a two-way Student's $t$ test. Values of $p<0.05$ were considered statistically significant.

Migration assay. Striata were removed from the embryos at $14.5 \mathrm{~d}$ after conception and mechanically dissociated as described previously (Lazarini et al., 2000). Briefly, migration of neural precursors was studied using a 48-well microchemotaxis chamber (Neuroprobe) and a poly-L-lysineprecoated membrane containing $8 \mu \mathrm{m}$ pores. Human SDF-1 $\alpha$ and its truncated form SDF-1 4-67 were a gift of Dr. F. Baleux (Pasteur Institute). For chemotaxis, SDF-1 $\alpha$ or SDF-1 4-67 was added in concentrations ranging from 2 to $800 \mathrm{~nm}$ in the bottom compartment, and $50 \mu \mathrm{l}$ of a $10^{6}$ cells $/ \mathrm{ml}$ suspension was added to the top compartment of each well. After $16 \mathrm{hr}$ incubation, the chamber was disassembled, and the cells that had migrated through the filter pores were fixed, stained, and counted as described previously (Lazarini et al., 2000). Results were expressed as mean \pm SD of cells migrating per four wells in five microscopic fields.

\section{Results}

\section{Identification of CXCR4 in Cajal-Retzius cells}

At the preplate stage of neocortical development (E13.5), CXCR4-expressing cells were confined primarily to the early marginal zone (Fig. 1 $A, B$ ). The high CXCR4 expression levels in
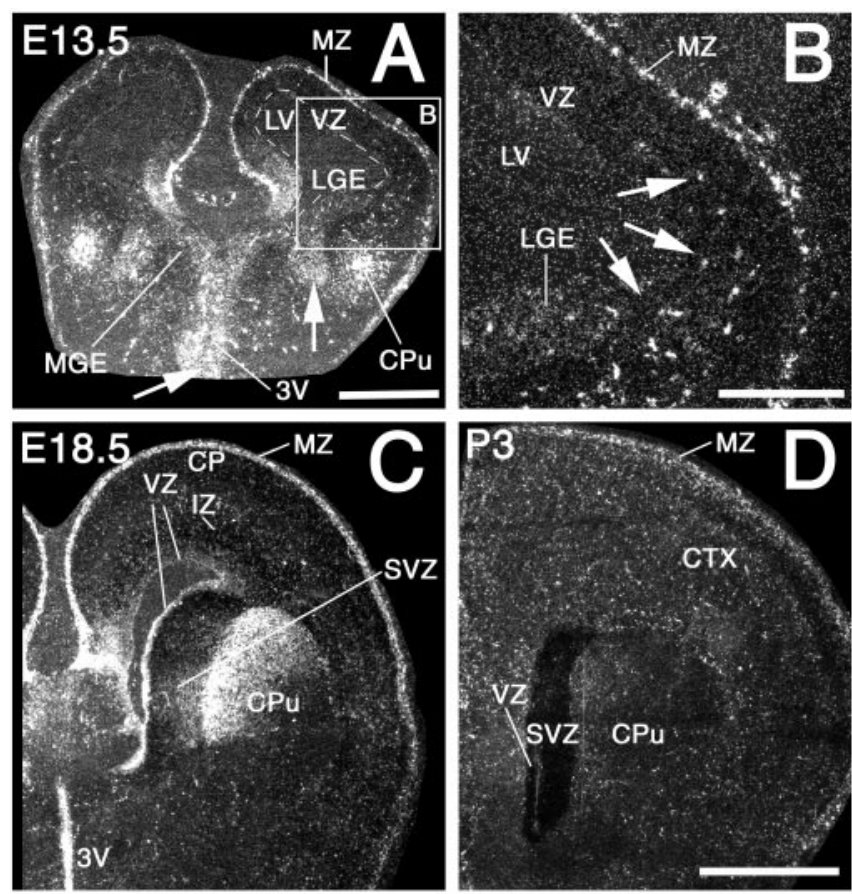

Figure 1. Regional distribution of CXCR4 mRNA in the developing forebrain. Dark-field micrographs of hybridized frontal sections. A, At E13.5 CXCR4 mRNA is expressed in single cells of the early marginal zone (MZ). Numerous CXCR4-expressing cells are detected in the germinal zones of the medial and lateral ganglionic eminences and preoptic area (arrows) as well as in the caudate-putamen (CPu). B, Note that CXCR4-expressing cells are distributed across the corticostriatal boundary (arrows). CXCR4 mRNA expression is absent from the ventricular germinal zone of the neocortex (VZ). C, At E18.5, CXCR4 mRNA is abundantly expressed in the neocortical marginal zone and the adjacent layer of the cortical plate (CP). CXCR4-expressing cells are present in all cortical layers including the intermediate zone (IZ) and the germinal zone below. Note the high CXCR4 expression in the subventricular (SVZ) and ventricular zones of the basal forebrain and in the caudate-putamen. D, At P3 high numbers of CXCR4-expressing cells are present in all layers of the cortex (CTX). CXCR4 expression in the caudate-putamen is strongly reduced as compared with $\mathrm{E} 18.5$. 3V, Third ventricle; LV, lateral ventricle; LGE and MGE, lateral and medial ganglionic eminence. Scale bars: $A, 0.6 \mathrm{~mm} ; B, 0.3 \mathrm{~mm} ; C, D, 1.5 \mathrm{~mm}$.

the marginal zone were maintained throughout late embryonic (E18.5) and early postnatal (P3) development (Figs. 1C,D, $2 B, C$, $3 A-D)$. During the first 3 postnatal weeks, the density of CXCR4 mRNA-containing neurons in the marginal zone/layer I decreased progressively (Fig. $4 E-H$, arrowheads). At E15.5 and P0, the vast majority of reelin-expressing neurons in the neocortical marginal zone contained CXCR4 mRNA, suggesting that CXCR4 is expressed in CR cells (Fig. 3E, representative image of E15.5). Thereafter, the proportion of CXCR4/reelin mRNA-expressing neurons from all reelin mRNA-expressing neurons in layer I decreased steadily (P6, 82\%; P9, 56\%; P12, 40\%; P21, 21\%; P31, $13 \%)$. The fact that a subpopulation of CXCR4-expressing cells did not contain reelin mRNA (E15.5, 25\%; P0, 43\%) indicates that CXCR4 is not selectively expressed in CR cells. Dual immunofluorescence revealed that reelin/CXCR4-immunoreactive (ir) CR cells exhibited a fusiform morphology with one thick unipolar dendrite that was tangentially orientated to the pial surface (Fig. 5A-C, arrowheads). Both cell body and dendrite exhibited numerous CXCR4-ir processes that extended perpendicularly to the pial surface (Fig. $5 A, C$ ). CR cells gave rise to CXCR4-ir axons (Fig. $5 D$, arrow) that contributed to a dense network of CXCR4-ir fibers in the deep aspect of layer I (Fig. 5C). Immunoreactive CXCR4 receptors were targeted predominantly to the plasma membrane (Fig. 5D). In layer I of the adult cerebral cortex, 

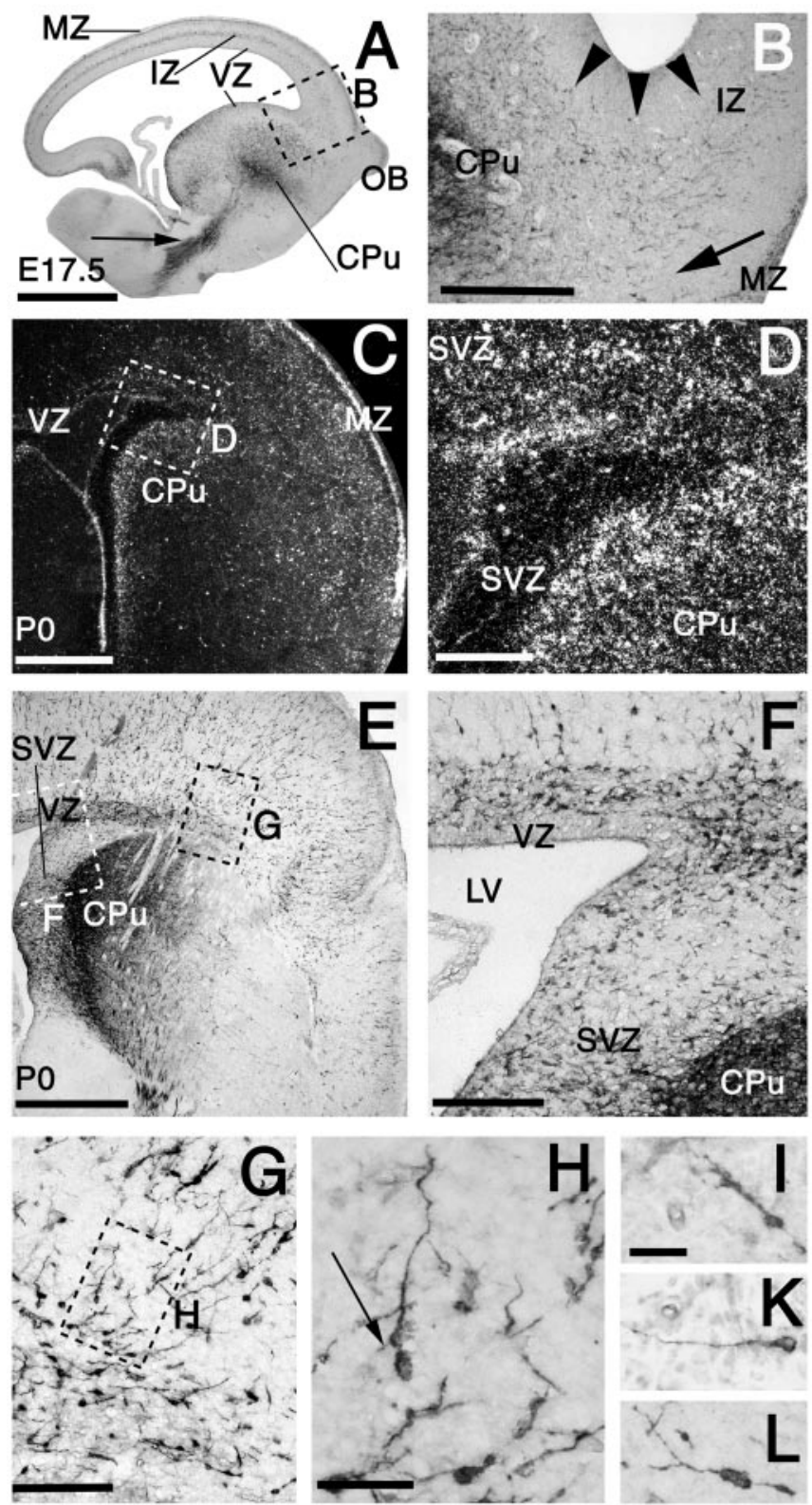

Figure 2. Distribution and morphology of CXCR4-expressing cells in the late embryonic and perinatal forebrain. $A$, Immunostained sagittal brain section (E17.5) showing XXCR4-LIR in the neocortical marginal and intermediate zones (MZ, IZ), olfactory bulb (OB), caudate-putamen $(\mathrm{CPu})$, and hippocampal anlage. Note the presence of $\mathrm{CXCR4-LIR} \mathrm{in} \mathrm{the} \mathrm{periventricular} \mathrm{zone} \mathrm{of}$ the basal forebrain and the absence of staining from the ventricular zone (VZ) of the neocortex. CXCR4-LIR in striatomesencephalic fibers (arrow) indicates that CXCR4 is also expressed in striatal projection neurons. $B$, Higher magnification of the transition zone of the basal forebrain and neocortex showing a stream of $C X C R 4$-ir neurons connecting the basal forebrain to the cortical intermediate zone (arrowheads) and the marginal zone (arrow). $C-F$, Comparison of in situ hybridization $(C, D$, coronal section) and immunohistochemistry $(E, F$, sagittal section) reveals virtually identical patterns of CXCR4 mRNA-expressing and CXCR4-ir cells in the PO forebrain. Note presence of CXCR4-expressing cells in all layers of the cortex including the ventricular and subventricular zones (VZ, SVZ), the medial aspect of the caudate-putamen, and the ventricular and subventricular zones of the basal forebrain. $G$, Distribution of tangentially orientated CXCR4-ir cells across the corticostriatal boundary. $H$ (arrow), XXCR4-ir neuron in the deep intermediate zone displaying morphological characteristics of a radially migrating neuron. Note that CXCR4-LIR is confined to the leading process and the thickened growth cone-like tip. I-L, Highpower magnification showing tangentially orientated CXCR4-ir neurons located at the corticostriatal boundary $(I, E 17.5)$, in the intermediate zone $(K, E 18.5)$, and in the cortical plate ( $L$, E18.5). Note intense $C X C R 4-L I R$ in the leading processes. Scale bars: $A, 0.8 \mathrm{~mm} ; B, 0.3 \mathrm{~mm} ; C, 1.0$ $\mathrm{mm} ; D, 0.3 \mathrm{~mm} ; E, 0.7 \mathrm{~mm} ; F, 0.3 \mathrm{~mm} ; G, 0.4 \mathrm{~mm} ; H, 30 \mu \mathrm{m} ; I-L, 30 \mu \mathrm{m}$.
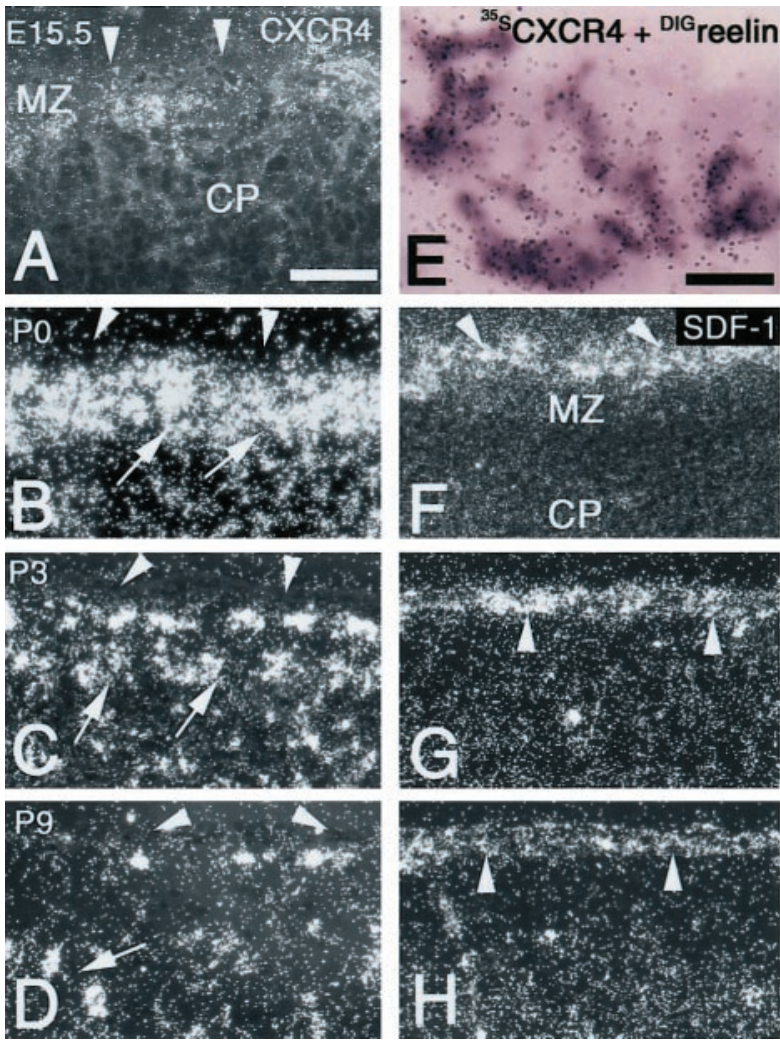

Figure 3. CXCR4 expression in the embryonic and postnatal marginal zone of the neocortex. Dark-field micrographs of the superficial layers of the neocortex after in situ hybridization for CXCR4 $(A-D)$ and SDF-1 $(F-H)$. In $A-D$ and $F-H$, the leptomeninx is indicated by arrowheads for orientation. $A$, At E15.5 CXCR4 mRNA is expressed in the marginal zone (MZ) but sparsely in the cortical plate (CP). $B-D$, The highest density of $C X C R 4$-expressing cells in the marginal zone is seen at $P 0$. From $P 0$ to $P 9$ the number of $C X C R 4$-expressing cells decreased progressively. $B-D$ (arrows), Note that numerous CXCR4-expressing cells are present at the border of the cortical plate and the marginal zone. F, At PO SDF-1 mRNA is highly expressed in meningeal cells. $G, H$, Meningeal SDF- 1 expression decreases progressively between $\mathrm{P} 3$ and P9. $F-H$, Note that SDF- 1 expression is virtually absent from cells in the marginal zone from $\mathrm{PO}$ to $\mathrm{P} 9$. E, Coexpression of CXCR4 and reelin mRNAs in cells of the E15.5 marginal zone shown by simultaneous in situ hybridization with a ${ }^{35}$-labeled probe for $\mathrm{CXCR4}$ (grains) and a digoxigenin-labeled probe for reelin (color precipitate). Scale bars: $A-D, F-H, 300 \mu \mathrm{m} ; E, 50 \mu \mathrm{m}$.

CXCR4-ir cells with CR cell-like morphology were detected only occasionally (Fig. 5E).

Next we analyzed the developmental expression of SDF-1. At embryonic and perinatal stages, SDF-1 is expressed at high levels in meningeal cells in juxtaposition to the CXCR4-containing neocortical marginal zone neurons (Figs. 3F-H, $4 I$, arrowheads). In contrast, SDF-1 was virtually absent from the marginal zone itself. Thus, meningeal cells are the most likely paracrine source providing SDF-1 for CXCR4-expressing marginal zone neurons in the developing neocortex. Postnatally, meningeal SDF-1 expression levels decreased in parallel to declining numbers of CXCR4-expressing cells in the marginal zone (Fig. $4 E-L$, arrowheads). Like that seen in the neocortex, the marginal zones of the dentate gyrus and hippocampus were transiently covered by cells expressing high SDF-1 levels (Fig. 4I-L, arrows) (Bagri et al., 2002; Lu et al., 2002) and exhibited a striking codistribution of CXCR4- and reelin-expressing cells (Fig. $4 A-H$, arrows). Unlike that observed in the neocortex, numerous hippocampal marginal zone neurons continued to express CXCR4 until P21 and adulthood (Stumm et al., 2002) (Fig. 4E-H, arrows). Although reelin mRNA was detected in the majority of CXCR4-expressing hip- 

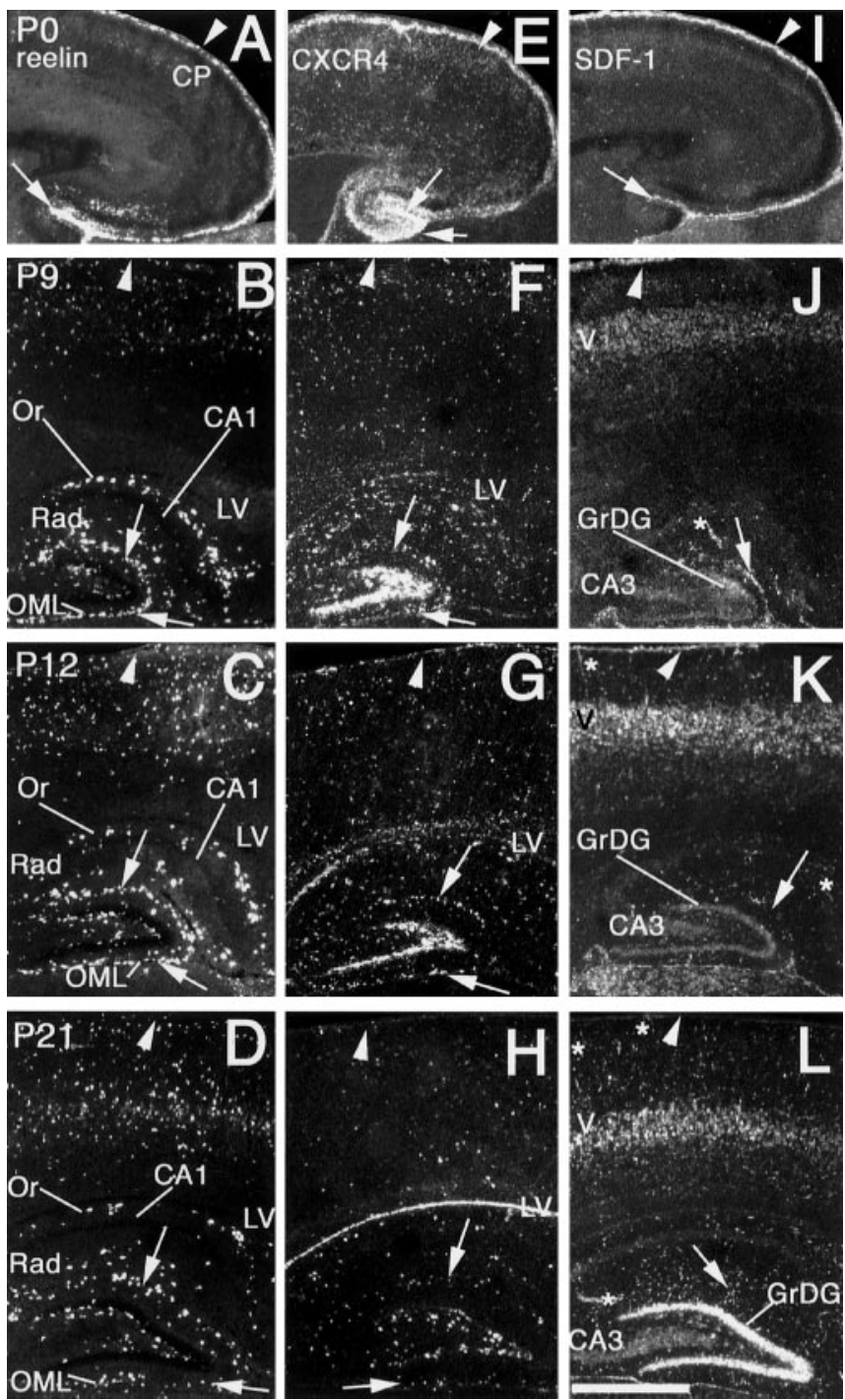

Figure 4. Comparison of postnatal development of reelin $(A-D)$, CXCR4 $(E-H)$, and SDF-1 $(I-L)$ mRNA expression in the neocortex and hippocampus. $A-L$, Dark-field micrographs of sagittal brain sections. A, E, At P0 both reelin- and CXCR4 mRNA-expressing cells are highly abundant in cortical layer I (arrowheads) and the outer molecular and lacunosum moleculare layers of the hippocampus (arrows). Note that CXCR4 is also expressed in the hippocampus and dentate gyrus. Numerous reelin-expressing cells are seen in the cortical plate (CP).I, At POSDF-1 mRNA is highly expressed in the leptomeninx (arrowhead) and the hippocampal fissure (arrow). $B-D$, From $P 9$ to P21 numerous reelin-expressing cells are detected in layer I (arrowheads), outer molecular layer (OML), lacunosum moleculare layer (located next to $\mathrm{OML}$ ), and oriens layer (Or) as well as the cortical layers II-VI. F, At P9 a high degree of codistribution of CXCR4- and reelin-expressing cells is observed in the oriens layer, stratum radiatum, moleculare lacunosum, and outer molecular layers. G, H, From P12 to P21 CXCR4 expression is progressively decreased in the forebrain. Note that high numbers of CXCR4-expressing cells are maintained in the outer molecular and lacunosum moleculare layers of the hippocampus (arrows). The high CXCR4 expression in granule cells of the dentate gyrus seen from P0 to P9 progressively declined to P21. Note that at P21 CXCR4 expression is virtually absent from granule cells, whereas CXCR4 is present in hilar neurons and neurons of the subgranular layer. From P9 to P21 CXCR4 expression in the ependymal layer of the lateral ventricle (LV) is increased. I-L, From P0 to P21 SDF-1 mRNA levels in the leptomeninx (arrowheads) and hippocampal fissure (arrows) decrease, whereas SDF-1 expression in cortical layer V (V) and pyramidal cell layer of the CA3 subfield of the hippocampus (CA3) as well as the granular layer of the dentate gyrus (GrDG) is increased. Note the increasing number of SDF-1expressing vascular structures (asterisks) after P9. Scale bar: (in $L$ ) $A-L, 1 \mathrm{~mm}$.

pocampal marginal zone neurons, GAD mRNA was undetectable in these neurons. Finally, these neurons displayed unique CR cell-like morphology, indicating that CXCR4 is expressed in hippocampal CR cells.
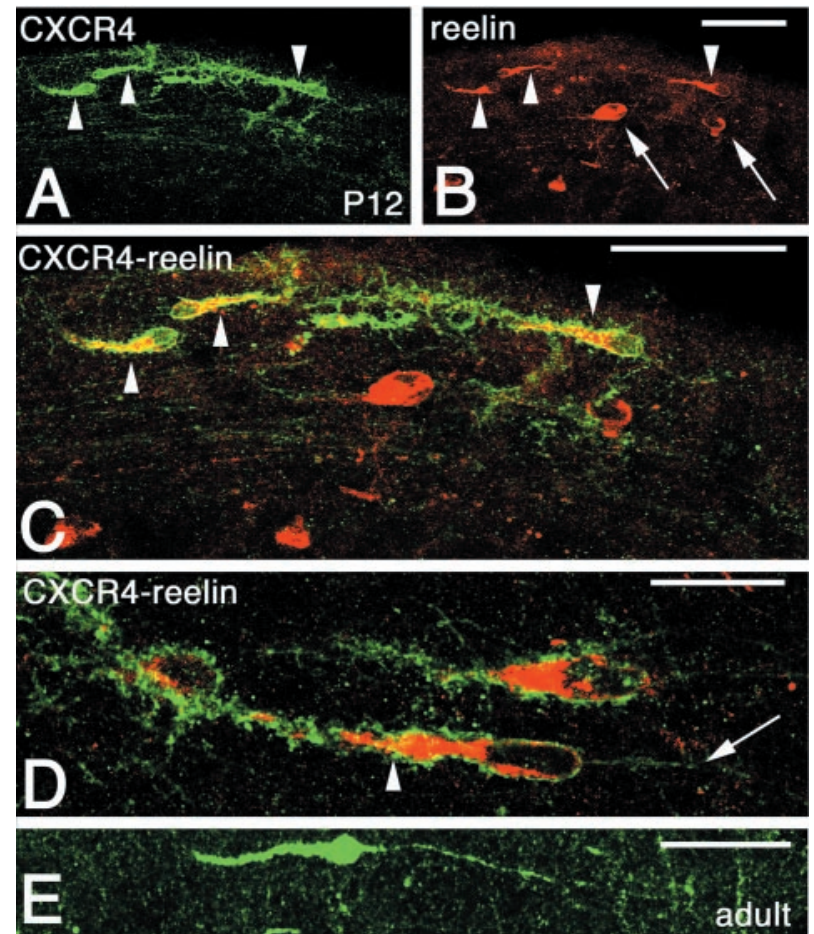

Figure 5. Identification of CXCR4-LIR in reelin-expressing CR cells in the marginal zone of the cerebral neocortex. $A-D$, Confocal images of the P12 cortical plate labeled for CXCR4 (green) and reelin (red) by dual immunofluorescence. $B$, Reelin-LIR is confined to CR cells in the marginal zone that are identified by their single large dendrite and parallel orientation to the meningeal surface (arrowheads). Reelin-LIR is also detected in interneurons in the cortical plate (arrows). A, C, CXCR4-LIR is detected in reelin-ir CR cells (arrowheads). Note that CXCR4-LIR is present in unique somatodendritic processes of $C R$ cells that run perpendicularly to the meningeal surface. CXCR4-ir axons arising from CR cells form a dense network in the deeper aspect of the marginal zone. $D$, Single confocal section demonstrating cytoplasmic localization of reelin and targeting of CXCR4 to the plasma membrane of soma and dendrite (arrowhead) as well as axon (arrow) of a CXCR4/reelin-ir CR cell. E, CR cell-like morphology of a CXCR4-ir neuron in the cerebrocortical layer I of an adult animal. Scale bars: $A-C, 80 \mu \mathrm{m} ; D, 25 \mu \mathrm{m} ; E, 50 \mu \mathrm{m}$.

Invasion of the cerebral cortex by CXCR4-expressing basal forebrain neurons

During late embryonic development (E17.5), numerous CXCR4expressing neurons emerged in the intermediate zone (Figs. $1 C$, $2 A, B)$ and at the border between cortical plate and marginal zone (Fig. $3 A, B$ ). In contrast, CXCR4-expressing cells were completely absent from the neocortical subventricular and ventricular germinal zones at E13.5 but emerged at E18.5 (Fig. $1 A-C$ ). In the basal forebrain, however, CXCR4 was transiently expressed at high levels in the germinal zones of the ganglionic eminences and in the caudate-putamen from E13.5 to P0 (Figs. 1, 2). In addition, numerous CXCR4-expressing cells were distributed across the corticostriatal boundary (Figs. 1B, 2A,B). Given that the basal forebrain is the origin of a large proportion of cerebrocortical interneurons, these findings are consistent with migration of CXCR4-expressing cells from the ganglionic eminences toward the cerebral cortex. Accordingly, CXCR4-ir cells at the corticostriatal boundary exhibited morphological characteristics resembling that of tangentially migrating neurons (Fig. 2I). These neurons had a prominent CXCR4-ir leading process and formed a bifurcated migratory stream connecting the ganglionic eminences to the intermediate zone and the border between marginal zone and cortical plate (Fig. 2B). During late embryonic development (E18.5-P0), tangentially oriented neurons were frequently detected in the intermediate zone and the periventricular 


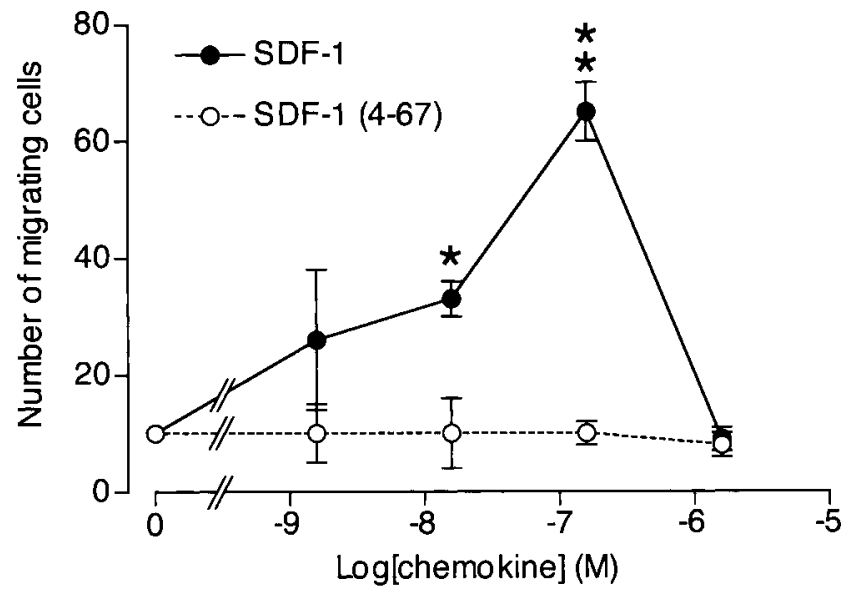

Figure 6. SDF-1 is a potent chemoattractant for striatal neuronal precursors. Shown is the dose-dependent migratory response of freshly isolated E14 striatal neural precursors to either SDF-1 or SDF-1 (4-67) after $16 \mathrm{hr}$ in chemotactic chambers (mean \pm SD of 3 experiments performed in quadruplicate). The peak migratory response occurred at $200 \mathrm{~nm}$ SDF-1. The truncated SDF-1 (4-67) does not evoke migratory responses. ${ }^{*} p<0.005,{ }^{* *} p<0.001$ significant difference between SDF-1 and SDF-1 (4-67); Student's $t$ test.

area (Fig. $2 K, L$ ). In addition, radially orientated CXCR4-ir neurons were seen from E18.5 to P0 throughout the cerebral cortex (Fig. 2E, $G, H$ ). Radially orientated neurons exhibited a large CXCR4-ir leading process with a thickened growth cone-like tip (Fig. $2 H$ ).

To determine whether SDF-1 provides a directional guidance cue for cerebrocortical interneuron precursors, we performed migration assays using freshly dissociated E14.5 mouse striata. As depicted in Figure 6, striatal neuronal precursors exhibited a robust and dose-dependent chemotactic response to SDF-1 but not to the truncated SDF-1 (4-67), which has a deletion of the three first amino acids in the $\mathrm{N}$ terminal extremity. SDF-1 (4-67) has previously been shown to be inactive when tested on lymphocytes in vivo and in vitro (Petit et al., 2002; Valenzuela-Fernandez et al., 2002). Neural precursors migrated toward SDF-1 only in response to a chemical gradient because they did not show enhanced migration when $200 \mathrm{~nm}$ of SDF-1 was added to the top and bottom compartments of the chemotactic chamber (data not shown).

In the cortical plate (layers II-VI), reelin is selectively expressed in interneurons (Alcantara et al., 1998; Pesold et al., 1998). At birth (P0), 78\% of reelin-expressing cortical plate neurons contained CXCR4 mRNA. The proportion of CXCR4/ reelin-expressing neurons from all CXCR4-expressing cells in cortical layers II-VI increased from $18 \%$ at P0 to $80 \%$ at P31, indicating that CXCR4 expression in the neocortex becomes confined primarily to interneurons with increasing age. This conclusion is supported by the finding that GAD mRNA was detectable in a vast majority of postnatal cerebrocortical CXCR4-expressing cells (data not shown). During the first 3 postnatal weeks, CXCR4 expression decreased strongly in most neocortical cells, including interneurons (Fig. $4 E-H$ ). Interestingly, the downregulation of CXCR4 expression in interneurons was paralleled by an upregulation of SDF-1 in pyramidal cells (Fig. $4 I-L$ ).

\section{Aberrant localization of reelin-expressing interneurons in CXCR4- and SDF-1-deficient mice}

To investigate the function of the SDF-1/CXCR4 system during development of the neocortex, we first analyzed CXCR4 expres-
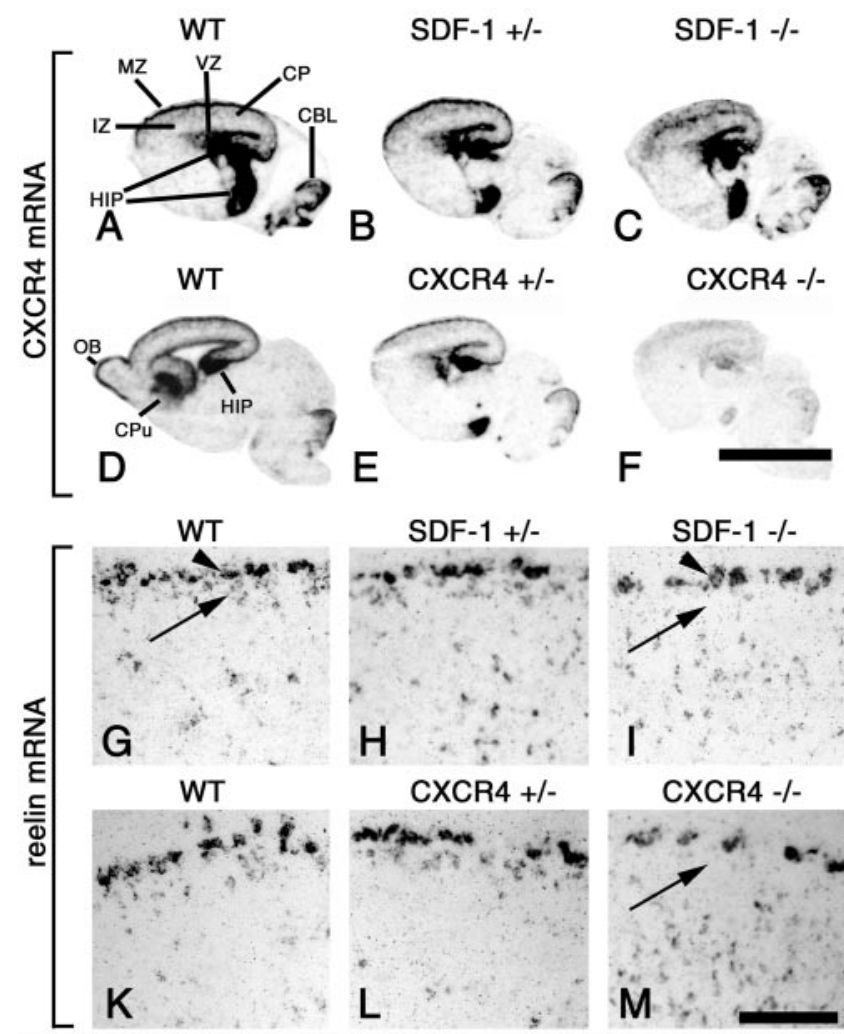

CXCR4 +/-

CXCR4 -/-
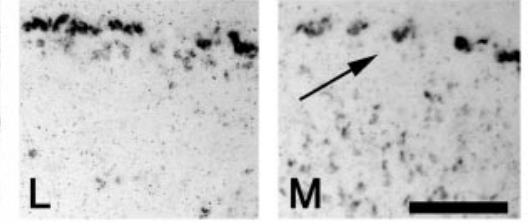

Figure 7. Aberrant patterning of $C X C R 4$ and reelin $m R N A$-expressing cells in the neocortex of E18.5 SDF-1- and CXCR4-deficient mice. $A-F$, Lateral sagittal brain sections hybridized for CXCR4 are shown. Hybridizations of wild-type mice (WT) are shown at both lateral $(A)$ and medial levels (D). $A, D$, Note that intense CXCR4 expression is seen in the marginal (MZ) and ventricular zones (VZ) of the neocortex, olfactory bulb (OB), caudate-putamen (CPu), hippocampus (HIP), and cerebellum (CBL) of wild-type mice. $A$, B, Similar CXCR4 expression patterns are observed in SDF- $1^{+1-}$ and wild-type mice. C, In SDF- $1^{-1-}$ mice, CXCR4 expression in the superficial layer of the neocortex is severely reduced, whereas ectopic CXCR4 expression is detected in the deeper aspect of the neocortex. $E$, $F$, Hybridization intensity is reduced in $\mathrm{CXCR}^{+1-}$ mice and at background level in CXCR4 ${ }^{-1-}$ mice, indicating specificity. $G-M$, Nonradioactive in situ hybridizations for reelin identifies large CR cells that express high levels of reelin mRNA ( $G$, arrowhead) and small reelin-expressing interneurons $(G$, arrow). Note that the positioning of $C R$ cells in the marginal zone is identical in all specimens. $I, M$, The number of $C R$ cells is slightly reduced in SDF- $1^{-1-}$ and CXCR4 ${ }^{-1-}$ mice. $G, H, K, L$, Reelin-expressing interneurons form a dense layer in the most superficial aspect of the cortical plate in wild-type and heterozygous mice (arrow). I, M, In SDF- $1^{-1-}$ and CXCR4 ${ }^{-1-}$ mice, the number of reelin-expressing interneurons in the superficial aspect of the cortical plate is severely reduced (arrows), whereas the number of reelin-expressing cells in the deeper aspect of the cortex is strongly increased. IZ, Intermediate zone of the neocortex. Scale bars: A-F, $6 \mathrm{~mm}$; $G-M, 250 \mu \mathrm{m}$.

sion in E18.5 SDF-1-deficient mice (Fig. $7 A-F$ ). The regional distribution of the CXCR4 mRNA in wild-type embryos is shown at a medial and a lateral sagittal section level (Fig. $7 A, D$ ). CXCR4 was highly expressed in the superficial layers and the ventricular zone of the neocortex, hippocampus, cerebellum, olfactory bulb, and caudate-putamen. This pattern was virtually identical in the SDF-1 heterozygous mice (Fig. 7 A,B). The specificity of hybridization is shown using CXCR4 heterozygous and CXCR4deficient mice (Fig. $7 E, F$ ). In the cortex of SDF-1-deficient mice, the strong CXCR4 expression in the superficial layers was severely reduced, and ectopic hybridization signals were observed in the deep aspect of the cortical plate and intermediate zone (Fig. 7C). Microscopic analysis revealed that the number of CXCR4expressing cells in the marginal zone and the adjacent layer of the cortical plate was severely reduced in the SDF-1-deficient mu- 

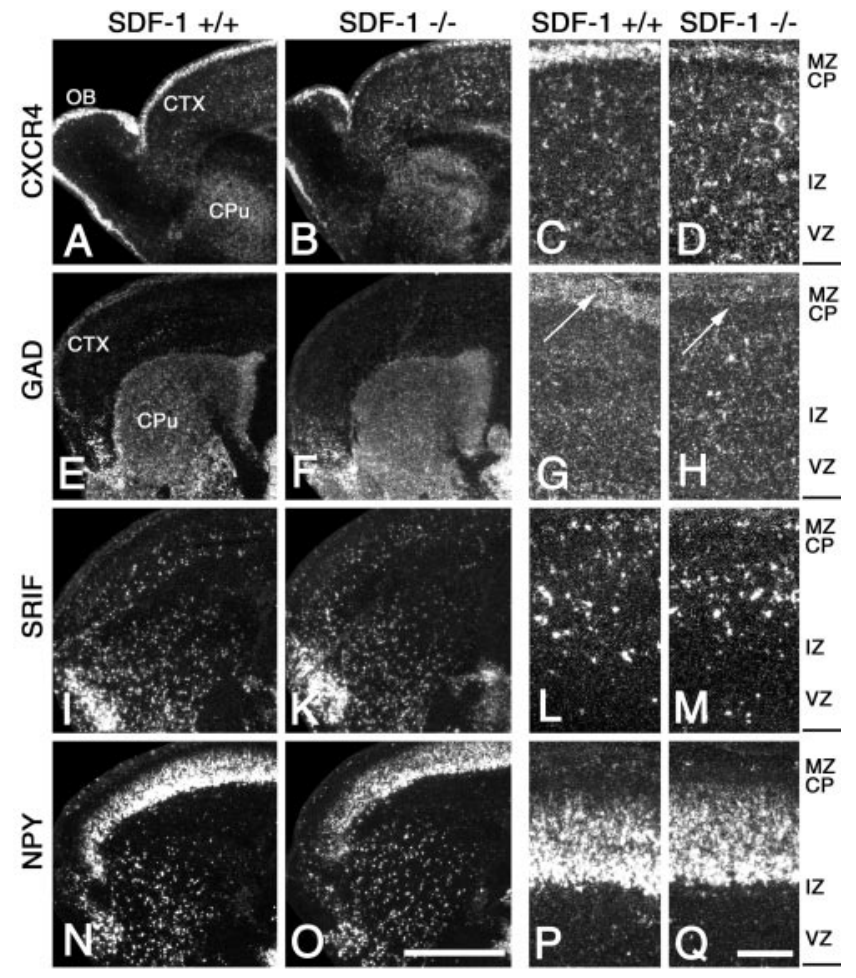

Figure 8. Patterns of CXCR4, glutamic acid decarboxylase (GAD), somatostatin (SRIF), and neuropeptide Y (NPY) expression in the forebrain of E18.5 SDF-1-deficient mice. $A, B, E, F, I, K, N$, 0 , Low-power dark-field micrographs of sagittal forebrain sections. $A, B$, Note that CXCR4expressing cells exhibit a similar distribution in the caudate-putamen (CPu) and across the corticostriatal boundary in SDF- ${ }^{+/+}$and SDF- $1^{-/-}$mice. The patterns of GAD $(E, F), \operatorname{SRIF}(I$, $K)$, and $\operatorname{NPY}(N, 0)$ expression in the caudate-putamen $(\mathrm{CPu})$ are virtually identical in SDF-1 ${ }^{+/+}$ and SDF-1 ${ }^{-1-}$ mice. $C, D, G, H, L, M, P, Q$, High-power dark-field micrographs of the neocortex (CTX).C, D, Note that the number of CXCR4-expressing cells at the border between cortical plate (CP) and marginal zone (MZ) is strongly reduced, whereas ectopic CXCR4 expression is seen in the deep aspect of the cortical plate and intermediate zone (IZ) in SDF-1 ${ }^{-1-}$ mice. In the superficial aspect of the neocortex of SDF- $1^{-1}$ mice, GAD mRNA levels are severely reduced $(G, H$, arrows), whereas SRIF mRNA levels are moderately reduced $(L, M, P, Q) . P, Q, N P Y$ expressing projection neurons in the cortical plate are distributed identically in SDF- $1^{+/+}$and SDF-1 ${ }^{-1-}$ mice. Scale bars: $A, B, E, F, I, K, N, 0,800 \mu \mathrm{m} ; C, D, G, H, L, M, P, Q, 250 \mu \mathrm{m}$.

tants (Fig. 8A-D). The number of CXCR4-expressing cells in the deep layers of the neocortex was increased in the SDF-1-deficient mice as compared with wild-type mice (Fig. $8 A-D$ ). In the basal forebrain, however, the pattern of CXCR4 and several markers for striatal projection and interneurons including GAD, somatostatin (SRIF), and neuropeptide Y (NPY) did not exhibit any obvious differences between SDF-1-deficient and wild-type mice (Fig. $8 A, B, E, F, I, K, N, O$ ). Given the extensive colocalization of CXCR4 and reelin in CR cells and cortical plate interneurons, we examined the distribution of reelin-expressing cells in the cortex of SDF-1- and CXCR4-deficient mice (Fig. 7G-M). The positioning in the marginal zone of CR cells, which are recognized by their characteristic large size and high reelin mRNA levels, was not altered in both null mutants as compared with wild-type mice (Fig. $7 G, K, I, M$, arrowheads). Morphometric analysis revealed a small reduction in the number of CR cells per area $(-26 \%$; $p<$ 0.05). A more pronounced effect was observed for reelinexpressing cortical plate neurons, which are smaller and exhibit considerably lower reelin mRNA levels than CR cells. These neurons formed a dense layer below the CR cells in wild-type mice and heterozygous mice but were completely absent from the superficial layers of the cortical plate in mice with a null mutation in
SDF-1 or CXCR4 (Fig. 7G-M, arrows). Conversely, in the deeper aspect of the neocortex the density of reelin-expressing interneurons was increased in these mice. In addition, the occurrence of GAD-expressing neurons was severely reduced in the superficial $(-55 \% ; p<0.05)$ and slightly increased in the deep aspect of the neocortex in both SDF-1 and CXCR4 null mutants as compared with wild-type mice (Fig. 8G,H). The number of SRIF-expressing neurons was also reduced $(-30 \% ; p<0.05)$ in the superficial part of the neocortex of SDF-1-deficient mice (Fig. $8 L, M$ ). In contrast to neocortical GABAergic neurons, the NPY-expressing projection neurons in the deep cortical plate exhibited virtually identical distribution patterns in null mutant and wild-type mice (Fig. 8N-Q). Together, these results strongly suggest that migration of GABAergic neocortical interneurons from the ganglionic eminences toward the superficial layers of the neocortex is severely impaired in mice with a defect in SDF-1/CXCR4 signaling. However, the migratory stream of CXCR4-expressing cells connecting the basal forebrain and the deep aspect of the neocortex was not disrupted in SDF-1-deficient mice (Fig. $8 A, B$ ), indicating that SDF-1 is not essential for CXCR4-expressing cells to reach the neocortex.

\section{Discussion}

In the present study we show that the spatial and temporal dynamics of CXCR4 expression in the developing embryonic forebrain are consistent with a role for SDF-1 in interneuron migration during development of the neocortex. The late-generated interneurons failed to integrate into the appropriate neocortical layer in the absence of functional SDF-1 signaling, whereas the positioning of early-generated preplate neurons in the marginal zone and the layering of projection neurons in the cortical plate were unaffected in mice lacking SDF-1 or CXCR4.

\section{SDF-1 is not required for positioning of CXCR4-expressing Cajal-Retzius cells}

CR cells are transient early-generated preplate neurons that are placed selectively in the outer marginal zone after development of the cortical plate (Super et al., 1998). We provide several lines of evidence that CXCR4 is expressed in CR cells. In the embryonic and perinatal marginal zones, the vast majority of reelin mRNAexpressing neurons contained CXCR4 mRNA. CXCR4expressing cells in the marginal zones were mostly GAD mRNA negative at P6 and P12. Furthermore, reelin/CXCR4-ir neurons in the marginal zones exhibited a typical fusiform CR cell-like morphology with one thick dendrite and numerous small somatodendritic processes. Finally, in the adult marginal zones CXCR4 was undetectable in the vast majority of reelin-expressing neocortical neurons but present in 30\% of reelin-expressing hippocampal neurons. These patterns are consistent with embryonic expression of CXCR4 in neocortical and hippocampal CR cells. These patterns are also consistent with a postnatal loss of neocortical CXCR4/reelin-expressing CR cells and the persistence of a significant proportion of CXCR4/reelin-expressing CR cells in the adult hippocampus (Alcantara et al., 1998; Super et al., 1998).

Given that CR cells extended somatodendritic processes particularly rich in CXCR4-like immunoreactivity (LIR) toward the SDF-1-expressing meningeal surface, we hypothesized that meningeal SDF-1 may act as a guidance cue or trophic factor for embryonic and perinatal CR cells. The latter idea is based on the observation that selective destruction of meningeal cells leads to secondary degeneration of CR cells, which indicates that meningeal cells synthesize and secrete trophic factors for CR cells (Super et al., 1998). Our observation that the number of CR cells is 
reduced by $26 \%$ in the neocortex in E18.5 SDF-1-deficient mice suggests that, in fact, SDF-1 may influence CR cell survival to some extent. In contrast, the submeningeal positioning of neocortical CR cells was not affected in SDF-1- or CXCR4-deficient mice. Thus, signaling of the CXCR4 receptor appears not to be involved in the positioning of early-generated neurons, which are thought to reach their final position by somal translocation (Nadarajah and Parnavelas, 2002).

\section{SDF-1 regulates migration of CXCR4-expressing neurons in the cortical plate}

A large proportion of cerebrocortical interneurons originates from the ventral forebrain (de Carlos et al., 1996; Anderson et al., 1997a,b; Nery et al., 2002). Neocortical interneurons initially migrate tangentially from the ganglionic eminences into the deep portion of the neocortex. Subsequently, these interneurons migrate radially toward the meningeal surface. A population of early-generated interneurons directly seeks the superficial aspect of the cortical plate (Marin and Rubenstein, 2001). We show that CXCR4 is present in neurons tangentially migrating from the basal forebrain toward the neocortex and that SDF-1 is a potent chemoattractant for isolated striatal neuronal precursors. In the cortical plate, the vast majority of reelin-expressing interneurons contained CXCR4, and CXCR4-expressing cells frequently coexpressed GAD. In both SDF-1- and CXCR4-deficient mice, reelin- and GAD-expressing interneurons were severely underrepresented in the superficial layers of the cortical plate but overrepresented in the deep aspect of the cortex. Thus, our findings strongly suggest that SDF-1, which is highly expressed in the embryonic leptomeninges, directly regulates migration of CXCR4-expressing interneurons from the deep portion of the neocortex toward the marginal zone. In addition, CXCR4expressing interneurons, which reach the marginal zone in wildtype mice, may integrate more often into the deeper part of the cortex in mice lacking SDF-1.

The expression patterns of CXCR4, GAD, SRIF, and NPY in the basal telencephalon and the transition zone between cortex and caudate-putamen did not show any obvious alterations in SDF-1-deficient mice, which is consistent with normal morphology of the basal ganglia in these mice (Ma et al., 1998). Thus, the absence of developmental defects from the basal ganglia and accumulation of numerous CXCR4/reelin-expressing cells in the deeper layers of the neocortex of SDF-1- and CXCR4-deficient mice indicates that tangential migration of striatal precursor cells from the ganglionic eminences to the neocortex does not require functional SDF-1/CXCR4 signaling. Recent evidence suggests that interneurons that are destined to populate the cortex express neuropilin 1 and neuroplinin 2, which make them responsive to a chemorepulsive signal provided by class 3 semaphorins in the striatal mantle. The chemorepulsive activity of these semaphorins is thought to channel striatal precursors into the tangential migratory stream directed toward the intermediate and marginal zones (Marin et al., 2001).

Little is known about factors that stop migration and induce differentiation of interneurons in the neocortex. Factors mediating arrest of migrating neural cells have been described recently in other CNS regions. Ephrin B2 can inhibit chemoattraction of cerebellar granule cells by meningeal SDF-1 during postnatal development (Lu et al., 2001). In the postnatal spinal cord, signaling by another chemokine receptor, CXCR2, has been shown to arrest oligodendrocyte migration in areas of localized expression of the CXCR2 ligand CXCL1 (Tsai et al., 2002). In contrast, Reelin may act as a dispersal factor rather than a stop signal for neural precursors to control layer organization (Hack et al., 2002). Our expression analysis of the postnatal cortex showed that the gradual upregulation of SDF-1 in pyramidal cells was paralleled by a progressive downregulation of CXCR4 in reelin- as well as GADexpressing interneurons. Thus, the postnatal induction of SDF-1 expression in principal neurons may be involved in the formation and refinement of local cerebrocortical circuits between pyramidal cells and immature interneurons that transiently express CXCR4

We have shown previously that severe ischemic damage of the cerebral cortex transiently downregulates SDF-1 and persistently upregulates CXCR4 in neurons of cortical areas surviving the insult indicating reactivation of the SDF-1/CXCR4 system under conditions of enhanced adult neurogenesis (Gu et al., 2000; Jiang et al., 2001; Lu et al., 2002; Stumm et al., 2002; Takasawa et al., 2002).

\section{Conclusion}

Taken together, our data provide the first evidence for a role of the SDF-1/CXCR4 receptor system in morphogenesis of the cerebral neocortex. We suggest that meningeal SDF-1 regulates radial migration of late-generated CXCR4-expressing interneurons in the cortical plate. In contrast, CXCR4-expressing preplate neurons do not appear to depend on SDF-1 for their early migration.

\section{References}

Alcantara S, Ruiz M, D’Arcangelo G, Ezan F, de Lecea L, Curran T, Sotelo C, Soriano E (1998) Regional and cellular patterns of reelin mRNA expression in the forebrain of the developing and adult mouse. J Neurosci 18:7779-7799.

Anderson SA, Eisenstat DD, Shi L, Rubenstein JL (1997a) Interneuron migration from basal forebrain to neocortex: dependence on Dlx genes. Science 278:474-476.

Anderson SA, Qiu M, Bulfone A, Eisenstat DD, Meneses J, Pedersen R, Rubenstein JL (1997b) Mutations of the homeobox genes Dlx-1 and Dlx-2 disrupt the striatal subventricular zone and differentiation of late born striatal neurons. Neuron 19:27-37.

Bagri A, Gurney T, He X, Zou YR, Littman DR, Tessier-Lavigne M, Pleasure SJ (2002) The chemokine SDF1 regulates migration of dentate granule cells. Development 129:4249-4260.

Bleul CC, Fuhlbrigge RC, Casasnovas JM, Aiuti A, Springer TA (1996) A highly efficacious lymphocyte chemoattractant, stromal cell-derived factor 1 (SDF-1). J Exp Med 184:1101-1109.

de Bergeyck V, Nakajima K, Lambert de Rouvroit C, Naerhuyzen B, Goffinet AM, Miyata T, Ogawa M, Mikoshiba K (1997) A truncated Reelin protein is produced but not secreted in the "Orleans" reeler mutation (Reln[rl-Orl]). Brain Res Mol Brain Res 50:85-90.

de Carlos JA, Lopez-Mascaraque L, Valverde F (1996) Dynamics of cell migration from the lateral ganglionic eminence in the rat. J Neurosci 16:6146-6156.

Doitsidou M, Reichman-Fried M, Stebler J, Koprunner M, Dorries J, Meyer D, Esguerra CV, Leung T, Raz E (2002) Guidance of primordial germ cell migration by the chemokine SDF-1. Cell 111:647-659.

Frotscher M (1998) Cajal-Retzius cells, Reelin, and the formation of layers. Curr Opin Neurobiol 8:570-575.

Gu W, Brannstrom T, Wester P (2000) Cortical neurogenesis in adult rats after reversible photothrombotic stroke. J Cereb Blood Flow Metab 20:1166-1173.

Haas CA, Deller T, Krsnik Z, Tielsch A, Woods A, Frotscher M (2000) Entorhinal cortex lesion does not alter reelin messenger RNA expression in the dentate gyrus of young and adult rats. Neuroscience 97:25-31.

Hack I, Bancila M, Loulier K, Carroll P, Cremer H (2002) Reelin is a detachment signal in tangential chain-migration during postnatal neurogenesis. Nat Neurosci 5:939-945.

Jiang W, Gu W, Brannstrom T, Rosqvist R, Wester P (2001) Cortical neurogenesis in adult rats after transient middle cerebral artery occlusion. Stroke 32:1201-1207

Klein RS, Rubin JB, Gibson HD, DeHaan EN, Alvarez-Hernandez X, Segal RA, Luster AD (2001) SDF-1 alpha induces chemotaxis and enhances 
Sonic hedgehog-induced proliferation of cerebellar granule cells. Development 128:1971-1981.

Lazarini F, Casanova P, Tham TN, De Clercq E, Arenzana-Seisdedos F, Baleux F, Dubois-Dalcq M (2000) Differential signaling of the chemokine receptor CXCR4 by stromal cell-derived factor 1 and the HIV glycoprotein in rat neurons and astrocytes. Eur J Neurosci 12:117-125.

Lu M, Grove EA, Miller RJ (2002) Abnormal development of the hippocampal dentate gyrus in mice lacking the CXCR4 chemokine receptor. Proc Natl Acad Sci USA 99:7090-7095.

Lu Q, Sun EE, Klein RS, Flanagan JG (2001) Ephrin-B reverse signaling is mediated by a novel PDZ-RGS protein and selectively inhibits $G$ proteincoupled chemoattraction. Cell 105:69-79.

Ma Q, Jones D, Borghesani PR, Segal RA, Nagasawa T, Kishimoto T, Bronson RT, Springer TA (1998) Impaired B-lymphopoiesis, myelopoiesis, and derailed cerebellar neuron migration in CXCR4- and SDF-1-deficient mice. Proc Natl Acad Sci USA 95:9448-9453.

Marin O, Rubenstein JL (2001) A long, remarkable journey: tangential migration in the telencephalon. Nat Rev Neurosci 2:780-790.

Marin O, Yaron A, Bagri A, Tessier-Lavigne M, Rubenstein JL (2001) Sorting of striatal and cortical interneurons regulated by semaphorinneuropilin interactions. Science 293:872-875.

Marin-Padilla M (1998) Cajal-Retzius cells and the development of the neocortex. Trends Neurosci 21:64-71.

Nadarajah B, Parnavelas JG (2002) Modes of neuronal migration in the developing cerebral cortex. Nat Rev Neurosci 3:423-432.

Nadarajah B, Brunstrom JE, Grutzendler J, Wong RO, Pearlman AL (2001) Two modes of radial migration in early development of the cerebral cortex. Nat Neurosci 4:143-150.

Nagasawa T, Hirota S, Tachibana K, Takakura N, Nishikawa S, Kitamura Y, Yoshida N, Kikutani H, Kishimoto T (1996) Defects of B-cell lymphopoiesis and bone-marrow myelopoiesis in mice lacking the CXC chemokine PBSF/SDF-1. Nature 382:635-638.

Nery S, Fishell G, Corbin JG (2002) The caudal ganglionic eminence is a source of distinct cortical and subcortical cell populations. Nat Neurosci 5:1279-1287.

Pesold C, Impagnatiello F, Pisu MG, Uzunov DP, Costa E, Guidotti A, Caruncho HJ (1998) Reelin is preferentially expressed in neurons synthesizing gamma- aminobutyric acid in cortex and hippocampus of adult rats. Proc Natl Acad Sci USA 95:3221-3226.

Petit I, Szyper-Kravitz M, Nagler A, Lahav M, Peled A, Habler L, Ponomaryov T, Taichman RS, Arenzana-Seisdedos F, Fujii N, Sandbank J, Zipori D, Lapidot T (2002) G-CSF induces stem cell mobilization by decreasing bone marrow SDF-1 and up-regulating CXCR4. Nat Immunol 3:687-694.

Stumm RK, Culmsee C, Schafer MK, Krieglstein J, Weihe E (2001) Adaptive plasticity in tachykinin and tachykinin receptor expression after focal cerebral ischemia is differentially linked to GABAergic and glutamatergic cerebrocortical circuits and cerebrovenular endothelium. J Neurosci $21: 798-811$.

Stumm RK, Rummel J, Junker V, Culmsee C, Pfeiffer M, Krieglstein J, Hollt V, Schulz S (2002) A dual role for the SDF-1/CXCR4 chemokine receptor system in adult brain: isoform-selective regulation of SDF-1 expression modulates CXCR4-dependent neuronal plasticity and cerebral leukocyte recruitment after focal ischemia. J Neurosci 22:5865-5878.

Super H, Soriano E, Uylings HB (1998) The functions of the preplate in development and evolution of the neocortex and hippocampus. Brain Res Brain Res Rev 27:40-64.

Super H, Del Rio JA, Martinez A, Perez-Sust P, Soriano E (2000) Disruption of neuronal migration and radial glia in the developing cerebral cortex following ablation of Cajal-Retzius cells. Cereb Cortex 10:602-613.

Tachibana K, Hirota S, Iizasa H, Yoshida H, Kawabata K, Kataoka Y, Kitamura Y, Matsushima K, Yoshida N, Nishikawa S, Kishimoto T, Nagasawa $\mathrm{T}$ (1998) The chemokine receptor CXCR4 is essential for vascularization of the gastrointestinal tract. Nature 393:591-594.

Takasawa K, Kitagawa K, Yagita Y, Sasaki T, Tanaka S, Matsushita K, Ohstuki T, Miyata T, Okano H, Hori M, Matsumoto M (2002) Increased proliferation of neural progenitor cells but reduced survival of newborn cells in the contralateral hippocampus after focal cerebral ischemia in rats. J Cereb Blood Flow Metab 22:299-307.

Tashiro K, Tada H, Heilker R, Shirozu M, Nakano T, Honjo T (1993) Signal sequence trap: a cloning strategy for secreted proteins and type I membrane proteins. Science 261:600-603.

Tham TN, Lazarini F, Franceschini IA, Lachapelle F, Amara A, Dubois-Dalcq M (2001) Developmental pattern of expression of the alpha chemokine stromal cell-derived factor 1 in the rat central nervous system. Eur J Neurosci 13:845-856.

Tsai HH, Frost E, To V, Robinson S, ffrench-Constant C, Geertman R, Ransohoff RM, Miller RH (2002) The chemokine receptor CXCR2 controls positioning of oligodendrocyte precursors in developing spinal cord by arresting their migration. Cell 110:373-383.

Valenzuela-Fernandez A, Planchenault T, Baleux F, Staropoli I, Le-Barillec K, Leduc D, Delaunay T, Lazarini F, Virelizier JL, Chignard M, Pidard D, Arenzana-Seisdedos F (2002) Leukocyte elastase negatively regulates stromal cell-derived factor-1 (SDF-1)/CXCR4 binding and functions by amino-terminal processing of SDF-1 and CXCR4. J Biol Chem 277:15677-15689.

Zhu Y, Yu T, Zhang XC, Nagasawa T, Wu JY, Rao Y (2002) Role of the chemokine SDF-1 as the meningeal attractant for embryonic cerebellar neurons. Nat Neurosci 5:719-720.

Zou YR, Kottmann AH, Kuroda M, Taniuchi I, Littman DR (1998) Function of the chemokine receptor CXCR4 in haematopoiesis and in cerebellar development. Nature 393:595-599. 